\title{
Isolation and identification of phylloplane and endophytic fungi from Sapindus mukorossi
}

B.D. Varpe

\author{
Department of Botany, \\ S.S.G.M.College, \\ Kopargaon, AHMEDNAGAR (M.S.) INDIA \\ E-mail : babasahebvarpe1964@gmail.com
}

Received : 25.08.2020; Accepted : 22.09.2020

\begin{abstract}
Phylloplane biodiversity and endophytic fungi is considered one of the rich origins of novel biological activity compounds and high-level structural variation on the leaf surface. Plant leaves surface is a diverse terrestrial ecosystem, including filamentous fungi. This study aims to study the isolation and enumeration of Sapindus mukorossi phylloplane and endophytic fungal diversity. The Sapindus mukorossi isolated 14 fungal species from 9 genera of phylloplane and endophytic fungi. Cladosporium herbarum, Penicillium expansum, Fusarium oxysporum, Fusarium sp., Alternaria alternate, Collectotrichum orbiculare, Torulla herbarium, Epicoccum nigrum and Candida sp. as a phylloplane fungi. Aspergillus niger, A. flavus, Epicoccum nigrum. Penicillum digitatum, Penicillum sp. were identified as endophytic fungi.

\section{Introduction}

The surface of plant leaves is a diverse terrestrial ecosystem dominated by a number of micro-organisms including bacteria, filamentous fungi and yeast phylloplane fungi are the mycota growing on the leaf surface. Phylloplane fungi were poorly studied among endophytes, saprobes, and pathogenic fungi ${ }^{7}$. Sapindus mukorossi plants were chosen for inquiry as they were still used in conventional medicines as well as hair and fabric washing. Isolation and characterization of phylloplane and endophytic fungi were performed from medicinal plant $S$. mukorossi during this research.

Plant morphology of Sapindus mukorossi - (Soap berries / Indian soapberry) it's a Sapindaceae family tree species. The fruit is widely known in the genus Sapindus as Indian soapberry or washing nut. It is from India's western seaside Maharashtra, Konkan, and Goa. Sapindus mukorossi known as a 'Ritha tree' in India and Nepal. It is a deciduous tree developed in the Himalayan's lower foot slopes and midhills up to 4000 feet. It is endurant to moderate nutrient poor soil, could be planted around farmers home and one seedling could produce 30-35 of fruits per year ${ }^{3}$.

Medicinal and commercial value of Sapindus mukorossi-The estimation of the tree originates from its natural product, which can be utilized for some pharmacological and cleaning purposes. The cleanser contains a saponin compound with characteristic cleaning properties and can likewise be utilized as a chemical for hair, skin and garments. These saponins are additionally utilized as a bug spray to expel head lice from the scalp. The cleanser nut can likewise be utilized as a characteristic remedy for other medical problems, for example, headache treatment, cerebral pains and seed disposal of skin pollutions, pimples or dermatitis ${ }^{8}$. It has been discovered that cleanser nut concentrates can be utilized as natural surfactants to improve the portability of field oil ${ }^{1}$.

\section{Materials and Methods}

Sample collection- Leaves of S. mukorossi were collected in fresh condition from R.B. N. B. College campus, Srirampur, Dist.-Ahmednagar, (MS). Healthy and mature leaf samples were carefully collected in the sterilized polythene bag and did tight the mouth of the bag to avoid any inoculation in it and immediately brought to the laboratory and kept in room temperature for further experiments.

Isolation of Fungi- For the isolation of fungi from the surface of the leaf, the process of printing the leaf was adopted. In order to remove the endophytic fungi, the extracted stable leaves have been carefully washed to hot tap water. A fragment of $1 \mathrm{~cm}^{2}$ of leaf blade was cut out of the basal section of the leaves. The leaf surface was cleaned for 1 moment by 0.1 percent mercuric chloride and washed 10 seconds in sterile sifted water. For phylloplane fungi, the fragments of leaves without washing were placed directly on the CDA media and the moist chamber equidistantly. 
Identification of Fungi- Phylloplane and Endophytic Fungi were identified with the help of standard manuals ${ }^{2}$.

\section{Culture of fungi on CDA plates}

Three leaves fragments, were pressed equidistantly on the CDA petri dishes with the help of sterile forceps, and kept for 7 to 21-days for incubation. Similar assortments of plates for the development of fungal microorganisms were set in a damp chamber.

\section{Result and Discussion}

Throughout the study, a total of 14 fungal species of 09 genera were isolated and identified from the healthy leaf of Sapindus mukorossi using both the CDA and the moist chamber process. This plant was screened for the existence of phylloplane and endophytic fungi, of which 14 species belonging to 9 genera of phylloplane and endophytic fungi were isolated, and the CDA plate method and the moist chamber method were established. Phylloplane and endophytic fungi isolated from $S$. mukorossiare shown in Table-1 of the CDA plate system Aspergillus niger, A. Flavus, Penicillum digitatum, Epicoccum nigrum have been classified as endophytic fungi and Aspergillus niger, Candida sp., Cladosporium herbarum, Collectotrichum orbiculare, Fusarium oxysporum, Fusarium sp., Epicoccum nigrum, Penicillium expansum, Penicillum sp. Alternaria alternata, Torulla herbarium, were found as phylloplane fungi. The present analysis found that the CDA and the moist chamber

TABLE-1 : Fungi isolated by CDA plate and moist chamber methods from medicinal plant Sapindus mukorossi

\begin{tabular}{|c|c|c|c|c|c|}
\hline \multirow[t]{2}{*}{$\begin{array}{l}\text { S. } \\
\text { No. }\end{array}$} & \multirow[t]{2}{*}{ Name of the Fungi } & \multicolumn{2}{|c|}{$\begin{array}{c}\text { Czapek's Dox Agar } \\
\text { medium }\end{array}$} & \multicolumn{2}{|c|}{$\begin{array}{l}\text { Moist chamber } \\
\text { method }\end{array}$} \\
\hline & & Endo. & Ecto. & Endo. & Ecto. \\
\hline 1 & Aspergillus niger & + & + & + & + \\
\hline 2 & A. flavus & + & - & - & - \\
\hline 3 & Candidasp. & - & + & - & + \\
\hline 4 & Cladosporium herbarum & - & + & - & + \\
\hline 5 & Collectotrichum orbiculare & - & + & - & + \\
\hline 6 & Epicoccum nigrum & + & + & + & + \\
\hline 7 & Fusarium oxysporum & - & + & - & + \\
\hline 8 & Fusarium sp. & - & + & - & + \\
\hline 9 & Penicillium expansum & - & + & - & + \\
\hline 10 & Penicillium digitatum & + & - & + & - \\
\hline 11 & Penicillium sp. & + & - & + & - \\
\hline 12 & Alternaria alternata & - & + & - & + \\
\hline 13 & Alternaria sp. & - & + & - & + \\
\hline 14 & Torulla herbarium & - & + & - & + \\
\hline
\end{tabular}


approach were equally appropriate for the isolation of endophytic and phylloplane fungi. Most of the fungi isolated belonged to deuteromycetes and ascomycetes ${ }^{4,5,6}$. Fungi have been isolated as endophytic and phylloplane that lead to the development of bioactive compounds. Harboring the effect of these fungi on the bioactive compounds found in Sapindus mukorossi. While the present study deals with the diversity of fungal communities associated with the medicinal plant Sapindus mukorossi, actual diversity can depend on the method used for collecting and handling leaf samples, the size of the leaf fragments and the cultivation of the herb 5 .

\section{Conclusion}

In the present investigation isolation of 14 phylloplane and endophytic fungal species were recorded. Among which Aspergillus and Epicoccum were the most dominant fungal species in both CDA and moist chamber method.

\section{References}

1. Chhetri $A B$, Watts $K C$, Rahman MS, Islam, MR. Soapnut extract as a natural surfactant for enhanced oil recovery. Energy sources, Part A: Recovery, utilization and Environmental effects. 2009; 31(20) 1893-1903.

2. Ellis MB. More Dematiaceous Hyphomycetes. Commonwealth Mycological Institute, Kew, Surrey, England. 1976; 507.

3. https://en.m.wikipedia.org.

4. Lokare PD. Effect of Roadside Air Pollution on Phylloplane Fungal Diversity of Polyalthia longifolia Sonn. International Jr. Of Science and Engineering, Special issue A4. 2018; 69-72.

5. Nayak BK. Isolation and identification of Phylloplane and endophytic fungi from one ornamental plant, Mangifera indica. International Jr. Of technochem Research. 2015; 1(3):188-192.

6. Nayak, BK. Studies on endophytic fungal diversity from different leaf samples of Pongamiapinnata. International Jr.Medipharma Res. 2015; 1 : 134-138.

7. Prabakaran M, Merinal S, Panneerslvam, A. Investigation of Phylloplane mycoflora from some medicinal plants. European Jr. of experimental Biology. 2011; 1(2):219-225.

8. Upadhyay A, Singh DK. Pharmacological effect of Sapindus mukorossi. Revista do insttuto de medicina Tropical de sao Paulo. 2012; 54(5): 273-280. 